\title{
Article
}

\section{Comparison of prestellar core elongations and large-scale molecular cloud structures in the Lupus 1 region}

Poidevin, Frédérick, Ade, Peter A. R., Angile, Francesco E., Benton, Steven J., Chapin, Edward L., Devlin, Mark J., Fissel, Laura M., Fukui, Yasuo, Gandilo, Natalie N., Gundersen, Joshua O., Hargrave, Peter C., Klein, Jeffrey, Korotkov, Andrei L., Matthews, Tristan G., Moncelsi, Lorenzo, Mroczkowski, Tony K., Netterfield, Calvin B., Novak, Giles, Nutter, David, Olmi, Luca, Pascale, Enzo, Savini, Giorgio, Scott, Douglas, Shariff, Jamil A., Soler, Juan Diego, Tachihara, Kengo, Thomas, Nicholas E., Truch, Matthew D. P., Tucker, Carole E., Tucker, Gregory S. and Ward-Thompson, Derek

Available at https://clok.uclan.ac.uk/11723/

Poidevin, Frédérick, Ade, Peter A. R., Angile, Francesco E., Benton, Steven J., Chapin, Edward L., Devlin, Mark J., Fissel, Laura M., Fukui, Yasuo, Gandilo, Natalie N. et al (2014) Comparison of prestellar core elongations and largescale molecular cloud structures in the Lupus 1 region. Astrophysical Journal, 791 (1). p. 43. ISSN 0004-637X

It is advisable to refer to the publisher's version if you intend to cite from the work. http://dx.doi.org/10.1088/0004-637x/791/1/43

For more information about UCLan's research in this area go to http://www.uclan.ac.uk/researchgroups/ and search for <name of research Group>.

For information about Research generally at UCLan please go to http://www.uclan.ac.uk/research/

All outputs in CLoK are protected by Intellectual Property Rights law, including Copyright law. Copyright, IPR and Moral Rights for the works on this site are retained by the individual authors and/or other copyright owners. Terms and conditions for use of this material are defined in the policies page. 


\title{
COMPARISON OF PRESTELLAR CORE ELONGATIONS AND LARGE-SCALE MOLECULAR CLOUD STRUCTURES IN THE LUPUS I REGION
}

\author{
Frédérick Poidevin ${ }^{1,2,3}$, Peter A. R. Ade ${ }^{4}$, Francesco E. Angile ${ }^{5}$, Steven J. Benton ${ }^{6}$, Edward L. Chapin ${ }^{7}$, \\ Mark J. Devlin ${ }^{5}$, Laura M. Fissel ${ }^{8,9}$, Yasuo Fukui ${ }^{10}$, Natalie N. Gandilo ${ }^{8}$, Joshua O. Gundersen ${ }^{11}$, Peter C. Hargrave ${ }^{4}$, \\ JefFrey Klein ${ }^{5}$, Andrei L. Korotkov ${ }^{12}$, Tristan G. Matthews ${ }^{9,13}$, Lorenzo Moncelsi ${ }^{14}$, Tony K. MroczKowski ${ }^{14}$, \\ Calvin B. NetTerfield ${ }^{6,8}$, Giles NovaK ${ }^{9,13}$, David Nutter ${ }^{4}$, Luca Olmi ${ }^{15,16}$, Enzo Pascale ${ }^{4}$, Giorgio Savini ${ }^{1}$, \\ Douglas Scott ${ }^{17}$, Jamil A. SharifF ${ }^{8}$, Juan Diego Soler ${ }^{8,18}$, Kengo Tachihara ${ }^{10}$, Nicholas E. Thomas ${ }^{11}$, \\ Matthew D. P. Truch ${ }^{5}$, Carole E. Tucker ${ }^{4}$, Gregory S. TuCKer ${ }^{12}$, And Derek Ward-Thompson ${ }^{19}$ \\ ${ }^{1}$ UCL, KLB, Department of Physics \& Astronomy, Gower Place, London WC1E 6BT, UK \\ 2 Instituto de Astrofísica de Canarias, E-38200 La Laguna, Tenerife, Spain; fpoidevin@iac.es \\ ${ }^{3}$ Dept. Astrofísica, Universidad de La Laguna, E-38206 La Laguna, Tenerife, Spain \\ ${ }^{4}$ School of Physics and Astronomy, Cardiff University, Queens Buildings, The Parade, Cardiff CF24 3AA, UK \\ ${ }^{5}$ Department of Physics and Astronomy, University of Pennsylvania, 209 South 33rd Street, Philadelphia, PA 19104, USA \\ ${ }^{6}$ Department of Physics, University of Toronto, 60 St. George Street, Toronto, ON M5S 1A7, Canada \\ ${ }^{7}$ XMM SOC, ESAC, Apartado 78, E-28691 Villanueva de la Canãda, Madrid, Spain \\ ${ }^{8}$ Department of Astronomy and Astrophysics, University of Toronto, 50 St. George Street, Toronto, ON M5S 3H4, Canada \\ ${ }^{9}$ Department of Physics and Astronomy, Northwestern University, 2145 Sheridan Road, Evanston, IL 60208, USA \\ ${ }^{10}$ Department of Physics, Nagoya University, Chikusa-ku, Nagoya, Aichi 464-8601, Japan \\ ${ }^{11}$ Department of Physics, University of Miami, 1320 Campo Sano Drive, Coral Gables, FL 33146, USA \\ ${ }^{12}$ Department of Physics, Brown University, 182 Hope Street, Providence, RI 02912, USA \\ ${ }^{13}$ Center for Interdisciplinary Exploration and Research in Astrophysics (CIERA), Northwestern University, 2145 Sheridan Road, Evanston, IL 60208, USA \\ ${ }^{14}$ California Institute of Technology, 1200 East California Boulevard, Pasadena, CA 91125, USA \\ ${ }^{15}$ Physics Department, University of Puerto Rico, Rio Piedras Campus, Box 23343, UPR station, San Juan, PR 00931, USA \\ ${ }^{16}$ Osservatorio Astrofisico de Arcetri, INAF, Largo E. Fermi 5, I-50125 Firenze, Italy \\ ${ }^{17}$ Department of Physics and Astronomy, University of British Colombia, 6224 Agricultural Road, Vancouver, BC V6T 1Z1, Canada \\ ${ }^{18}$ CNRS-Institut d'Astrophysique Spatiale, Université Paris-XI, F-91405 Orsay, France \\ ${ }_{19}$ Jeremiah Horrocks Institute, University of Central Lancashire, PR1 2HE, UK \\ Received 2014 April 30; accepted 2014 June 24; published 2014 July 24
}

\begin{abstract}
Turbulence and magnetic fields are expected to be important for regulating molecular cloud formation and evolution. However, their effects on sub-parsec to 100 parsec scales, leading to the formation of starless cores, are not well understood. We investigate the prestellar core structure morphologies obtained from analysis of the Herschel-SPIRE $350 \mu \mathrm{m}$ maps of the Lupus I cloud. This distribution is first compared on a statistical basis to the large-scale shape of the main filament. We find the distribution of the elongation position angle of the cores to be consistent with a random distribution, which means no specific orientation of the morphology of the cores is observed with respect to the mean orientation of the large-scale filament in Lupus I, nor relative to a large-scale bent filament model. This distribution is also compared to the mean orientation of the large-scale magnetic fields probed at $350 \mu \mathrm{m}$ with the Balloon-borne Large Aperture Telescope for Polarimetry during its 2010 campaign. Here again we do not find any correlation between the core morphology distribution and the average orientation of the magnetic fields on parsec scales. Our main conclusion is that the local filament dynamics-including secondary filaments that often run orthogonally to the primary filament-and possibly small-scale variations in the local magnetic field direction, could be the dominant factors for explaining the final orientation of each core.
\end{abstract}

Key words: ISM: clouds - ISM: individual objects (Lupus I) - ISM: magnetic fields - polarization submillimeter: ISM

Online-only material: color figures

\section{INTRODUCTION}

Understanding the processes leading to the formation of stars in our Galaxy is one of the great challenges which, despite much progress (e.g., Molinari et al. 2014), still remains open. At subparsec scales, the detailed mechanisms remain elusive through which gravitational collapse occurs, leading to the formation of a prestellar core which eventually will give birth to one or more stars. Recent advances have shown that turbulence is a key ingredient and plays a dual role, both creating overdensities to initiate core formation and counteracting the effects of gravity into the denser regions of these objects (e.g., McKee \& Ostriker 2007). In addition to gravity and turbulence, other physical processes are likely to play a significant role. Specifically, magnetic field and dynamical chemistry networks are expected to be relevant for understanding the general phenomenology of star formation (see, for example, Leão et al. 2013; Girart et al. 2013; Tassis et al. 2012a, 2012b, 2012c, and references therein). However, all in all, it is currently unclear which of all these mechanisms dominates over the other ones, and over which spatial and temporal scales.

On larger spatial scales, the formation and evolution of molecular clouds is not well understood and there is abundant literature on the subject. In particular, several simulation approaches addressing these questions have been developed over the last two decades (e.g., Ostriker et al. 2001; Gammie et al. 2003; Falceta-Gonçalves et al. 2008; Heitsch et al. 2009; Nakamura \& Li 2011; Bonnell et al. 2013). While these analyses sometimes 
Table 1

Location of Cores and the Estimates of Their Position Angle Elongation EPA and Axis Ratio as Obtained from the Lorentzian Fitting Method Discussed in Section 4.1

\begin{tabular}{|c|c|c|c|c|c|}
\hline Index & $\begin{array}{c}\text { R.A. }(\mathrm{J} 2000) \\
\left(^{\circ}\right)\end{array}$ & $\begin{array}{c}\text { Decl. }(\mathrm{J} 2000) \\
\left({ }^{\circ}\right)\end{array}$ & $\begin{array}{c}\mathrm{EPA} \\
\left(^{\circ}\right)\end{array}$ & $\begin{array}{l}\text { Axis } \\
\text { Ratio }\end{array}$ & LPCP \\
\hline 1 & 234.597 & -34.8721 & 151 & 1.2 & 0.88 \\
\hline 2 & 234.837 & -34.7280 & 131 & 1.8 & 0.74 \\
\hline 3 & 235.030 & -33.5602 & 40 & 3.3 & 0.95 \\
\hline 5 & 235.524 & -34.1556 & 89 & 1.8 & 0.79 \\
\hline 6 & 235.547 & -34.1519 & 98 & 8.1 & 0.84 \\
\hline 8 & 235.578 & -33.8461 & 71 & 1.7 & 0.75 \\
\hline 9 & 235.654 & -33.8625 & 108 & 1.7 & 0.93 \\
\hline 10 & 235.706 & -33.9900 & 153 & 4.5 & 0.87 \\
\hline 12 & 235.729 & -34.0750 & 136 & 1.8 & 0.91 \\
\hline 13 & 235.817 & -34.0778 & 165 & 1.6 & 0.85 \\
\hline 15 & 236.029 & -34.6499 & 68 & 2.1 & 0.71 \\
\hline 16 & 236.171 & -34.3421 & 54 & 1.3 & 0.72 \\
\hline 17 & 236.183 & -34.2955 & 14 & 1.9 & 0.73 \\
\hline 18 & 236.247 & -34.2861 & 121 & 2.6 & 0.91 \\
\hline 19 & 236.300 & -34.2855 & 85 & 2.2 & 0.92 \\
\hline 20 & 236.325 & -34.2093 & 70 & 1.5 & 0.79 \\
\hline 25 & 236.485 & -34.4937 & 118 & 1.8 & 0.92 \\
\hline 26 & 236.566 & -34.5111 & 62 & 1.6 & 0.80 \\
\hline 27 & 236.631 & -34.5514 & 95 & 1.6 & 0.86 \\
\hline
\end{tabular}

Note. Also given in the last column is the linear Pearson coefficient parameter (LPCP) between model and data distributions.

use very different lines of reasoning, almost all of them include and/or show that the combined effects of magnetic fields and turbulence are key ingredients to understanding core mass function estimates for our Galaxy. However, the impact of magnetic fields on different spatial and density scales has not yet been established with regard to explaining the observed star formation rate (SFR). Recent work suggests that magnetic fields are regulating cloud formation (e.g., Heitsch et al. 2009; Nakamura \& Li 2011), with different scenarios depending on the magnetic field strength and orientation with respect to outflow-driven turbulence.

In practice, characterization of cloud structure properties and star formation efficiency based on map analysis show strong variations from one cloud to another (e.g., Schneider et al. 2013; Rygl et al. 2013). Recent studies may provide clues pointing toward the general mechanisms dominating the processes in different regions. In one such study, BallesterosParedes et al. (2011) propose that bound molecular clouds could be in a state of hierarchical and chaotic gravitational collapse. On the other hand, Poidevin et al. (2013) show that simple ideal, isothermal, and non-self gravitating MHD simulations are sufficient to describe the large-scale observed physical properties of the envelopes of four different molecular clouds. This result is consistent with some of the molecular clouds not being necessarily gravitationally bound as discussed by Ward et al. (2014). These findings raise important questions regarding the range of spatial scales and density regimes which are involved with non-self gravitating MHD and effective local collapse leading to core formation.

The Lupus I molecular cloud complex has already been well studied (e.g., Hara et al. 1999). We do not know if these clouds are bound or unbound. However, related to the questions above, one can study the relation between the large-scale structures of the filamentary molecular clouds and the average distribution of the prestellar core structure morphologies associated with that region. This is the central question addressed in this work.
We focus our study on the structural morphologies of the prestellar cores obtained from the analysis of the $350 \mu \mathrm{m}$ SPIRE images associated with these clouds (see Rygl et al. 2013). The morphology of the large-scale filamentary structure of Lupus I is discussed in a separate paper by Matthews et al. (2014). In addition to photometric imaging, we use the submillimeter polarimetry data set on Lupus I obtained from the 2010 Balloon-borne Large Aperture Telescope for Polarimetry (BLASTPol) flight (see Matthews et al. 2014). Ideally, it would be important to compare each identified core morphology with the local magnetic field. In practice, the resolution of the 2010 BLASTPol data precludes this approach, and only the average core morphology (taken to be the average orientation of the core long axes) can be compared to average direction of the cloud magnetic field. Therefore, we limit our analysis to these statistical properties.

In the following, Section 2 gives a brief description of the Lupus I region. The data used in this analysis are described in Section 3. We use the list of cores detected by Rygl et al. (2013) in the SPIRE data, and Section 4 discusses the methodology adopted to determine the average elongation of these sources. A comparison of the average filamentary molecular cloud structures and the sample of prestellar cores for which it is possible to define accurate average elongation position angle (EPA) is given in Section 5. A comparison of the mean magnetic field orientation and the prestellar core orientations is also given. Our results and their implication are discussed in Section 6 with conclusions provided in Section 7.

\section{THE LUPUS I REGION}

The Lupus I molecular cloud region is a well studied site of star formation. It is one of the closest star-forming regions at $(155 \pm 8) \mathrm{pc}$ (see Lombardi et al. 2008), lying close to the position centered at R.A. (J2000) $=15^{\mathrm{h}} 42^{\mathrm{m}} 00^{\mathrm{s}}$, decl. $(\mathrm{J} 2000)=-34^{\circ} 12^{\prime} 00^{\prime \prime}$. Using the existing Spitzer catalog and SPIRE imaging at 250, 350, and $500 \mu \mathrm{m}$, Rygl et al. (2013) detected cores located in this region and discuss their evolutionary classification. These authors show that Lupus I is undergoing a large star formation event, as estimated by the increased SFR and by the large number of prestellar objects when compared to more evolved structures. However, the mechanism behind this surge in star formation is not well understood.

\section{DATA SETS}

\subsection{Prestellar Core Sample}

In our analysis we use the sample of prestellar cores identified by Rygl et al. (2013) with their combined analysis of the Herschel 70-500 $\mu \mathrm{m}$ maps. The paper does not display an explicit list of coordinates of the cores they discuss. Therefore, for identifying the coordinates of the prestellar cores, we used the central position of the maps displayed in their Figure A.2. Most of the time the peak location of the core was obvious, being no further than a few arcseconds from the center of their map. In such cases we report these coordinates, and if not we report the center coordinates of their map. The intensity maps shown in our Figure 1 (discussed further below) compare well with the intensity maps shown by Rygl et al. (2013) and we believe our core position coordinate estimates are accurate to $\approx 10^{\prime \prime}$. These core position coordinate estimates are provided in Columns 2 and 3 of Tables 1 and 2. 

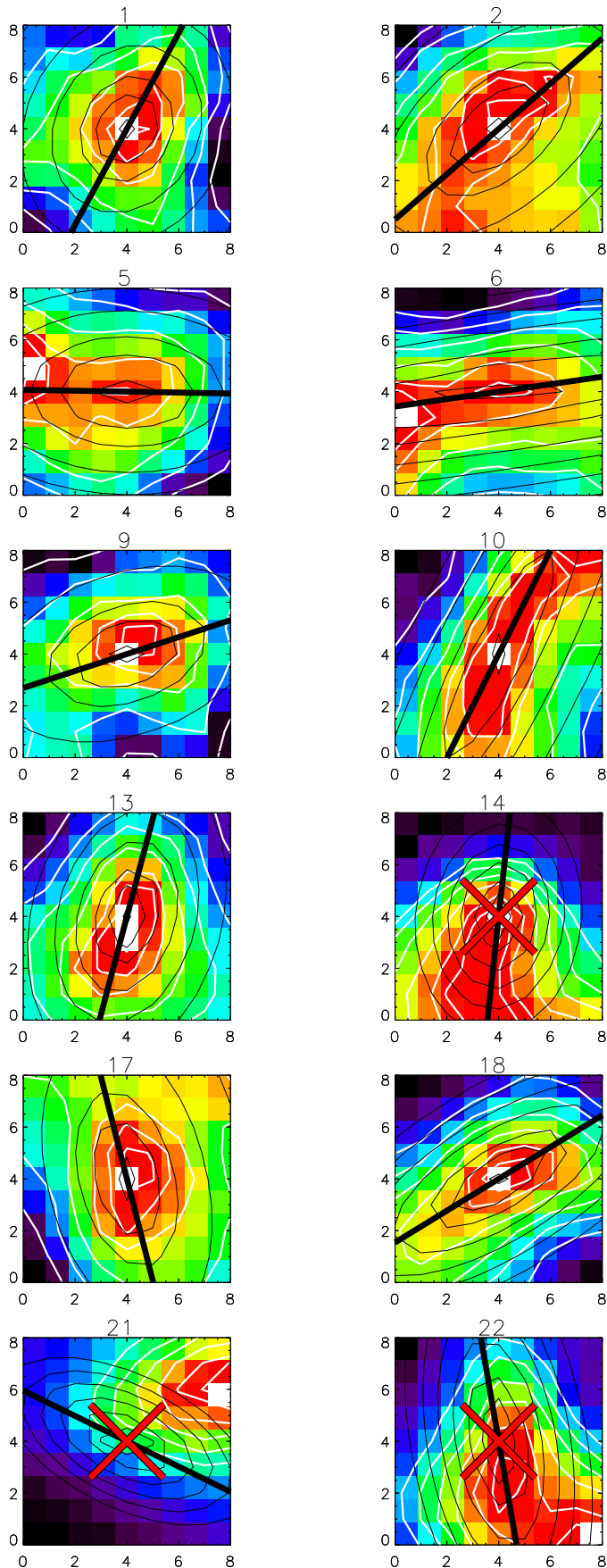

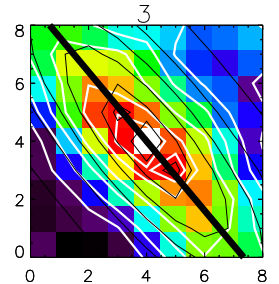

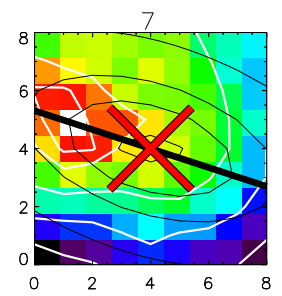
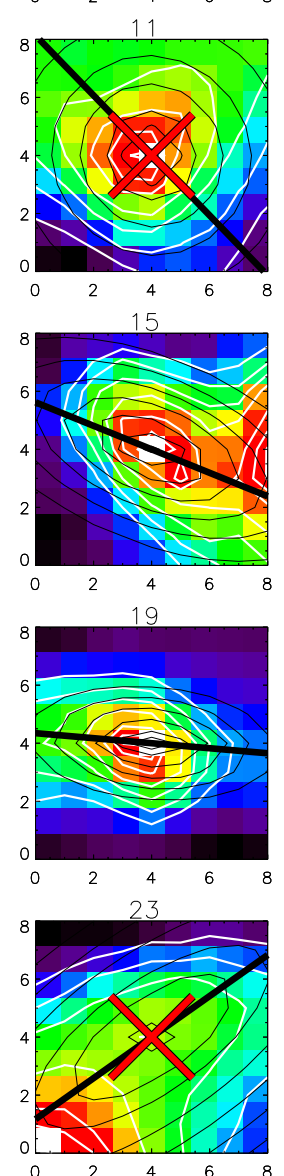
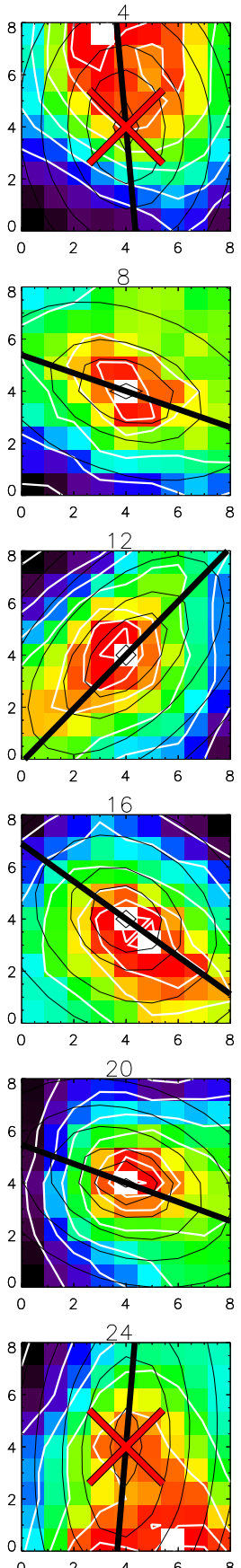
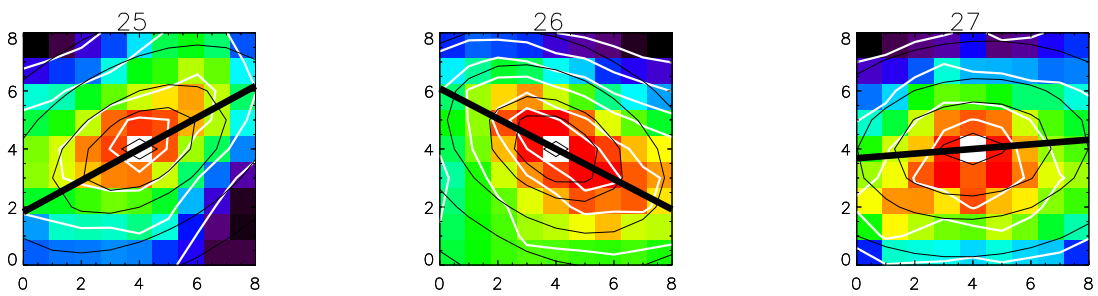

Figure 1. Snapshot of the intensity structures centered on the position of the cores listed in Tables 1 and 2 . The black line segment shows the elongation of the intensity structure obtained with the Lorentzian fitting method. White lines show intensity contours obtained at levels of $0.5,0.6,0.7,0.8,0.9,0.95$, and 0.99 of the peak. Dark lines show similar contour lines obtained from the Lorentzian fit models. The red crosses show the prestellar cores rejected from the later analysis, as discussed in Section 4.1.

(A color version of this figure is available in the online journal.)

\subsection{BLASTPol Polarimetry Data Set}

One of the best ways for probing magnetic fields in molecular clouds is through submillimeter (submm) polarimetry, where the radiation from aspherical dust grains, aligned by the local magnetic field (see Lazarian 2007, for a review), is detected in polarization. Therefore, submm polarimetry data provide information about the mean projected component of the magnetic 
Table 2

List of Cores Rejected from Our Analysis because We Cannot Reliably Estimate the EPA, as Discussed in Section 4.1

\begin{tabular}{lccccr}
\hline \hline Index & $\begin{array}{c}\text { R.A. }(\mathrm{J} 2000) \\
\left({ }^{\circ}\right)\end{array}$ & $\begin{array}{c}\text { Decl. }(\mathrm{J} 2000) \\
\left({ }^{\circ}\right)\end{array}$ & $\begin{array}{c}\text { EPA } \\
\left({ }^{\circ}\right)\end{array}$ & $\begin{array}{c}\text { Axis } \\
\text { Ratio }\end{array}$ & LPCP \\
\hline 4 & 235.042 & -34.9250 & $\ldots$ & 1.4 & 0.47 \\
7 & 235.565 & -33.8499 & $\ldots$ & 2.0 & 0.60 \\
11 & 235.704 & -34.2224 & $\ldots$ & 1.0 & 0.73 \\
14 & 236.000 & -34.6417 & $\ldots$ & 1.4 & 0.50 \\
21 & 236.325 & -34.2889 & $\ldots$ & 2.0 & 0.10 \\
22 & 236.342 & -34.2541 & $\ldots$ & 2.4 & 0.61 \\
23 & 236.353 & -34.3681 & $\ldots$ & 2.5 & 0.63 \\
24 & 236.367 & -34.3806 & $\ldots$ & 2.0 & 0.55 \\
\hline
\end{tabular}

Note. Information displayed is as in Table 1.

field on the plane of the sky (POS). We use BLASTPol (see Pascale et al. 2012) submm polarimetry to infer the POS magnetic field orientations.

Details about the BLAST and BLASTpol experiment, instruments, and flights are given by Pascale et al. (2012), Moncelsi et al. (2014), and F. A. Angilè et al. (2014, in preparation). Matthews et al. (2014) provides a detailed discussion of the polarimetry analysis of the 2010 BLASTPol data at 250, 350, and $500 \mu \mathrm{m}$. Most of the modified blackbody fits of the prestellar cores discussed by Rygl et al. (2013) peak (in $S_{v}$ units) at a wavelength close to $350 \mu \mathrm{m}$. For this reason, we focus our analysis on the polarimetry data set obtained at this wavelength. This data set is identical to the one used by Matthews et al. (2014) in their analysis.

\section{DATA ANALYSIS}

In this section the position angles (PA), whether they refer to core EPA as seen on the POS, or to mean magnetic field orientations, are counted positively from north in an anticlockwise direction.

The polarization PA is periodic and is defined to wrap around in a $\left[0^{\circ}, 180^{\circ}\right]$ period. The median values retained in our analysis correspond to the mean and median estimates obtained such that the dispersions of the distributions are found to be the smallest.

\subsection{Core Position Angle Estimates}

To define the averaged orientation on the POS of the elongation of each prestellar core structure, we use the Herschel $350 \mu \mathrm{m}$ intensity map. The spatial limit between the cores and the cloud envelopes in which they are embedded is not always clearly defined, because of the limit of the resolution of the map. Also dust grain emission along the line of sight (LOS) and the presence of other core structures can cause confusion. In their work, however, Rygl et al. (2013) consider a prestellar core to be defined as a gravitationally bound region of size $<0.05 \mathrm{pc}$. We adopt the spatial scale, $l_{\min }=0.05 \mathrm{pc}$, as the smallest that should be used for characterizing the prestellar core shapes. With a distance to Lupus I of $\approx 155$ pc (Lombardi et al. 2008) this means that in the Herschel $350 \mu \mathrm{m}$ intensity map, prestellar cores should be well sampled through kernels of $7 \times 7$ pixels with pixels of size $10^{\prime \prime} \times 10^{\prime \prime}$. To ensure that the structure of each prestellar core is fully included in our analysis, we have decided to use slightly larger kernels of $9 \times 9$ pixels for estimating the elongations of the observed morphologies of the cores. This ensures a large enough sample of pixels for the fitting procedure that we detail below, and also ensures direct comparison with the maps of the prestellar cores displayed by Rygl et al. (2013) in their Figure A.2.

Gravity is expected to be the dominant mechanism that shapes core morphologies. Therefore, we assume that the shape of a prestellar core can be modeled by a spheroid, and that the size and orientation of the main axis of this spheroid (after projection on the POS) can be approximated and described by the parameters associated with a two-dimensional (2D) Lorentzian distribution ${ }^{20}$ (see Johnstone et al. 2000; Kirk et al. 2006; Planck Collaboration 2011, and references therein for more details about clump characterizations with 2D Gaussian fitting methods). Estimates of core elongations have been obtained with the IDL mpfit $2 \mathrm{dpeak}^{21}$ routine assuming Lorentzian distributions. The fits have been obtained by only forcing the center of the Lorentzian models to peak at the position listed in Tables 1 and 2, and otherwise the remaining parameters (constant baseline level, peak value, half-width values along the short and long axis and position angle) were left free in the fit.

Snapshots of the selected prestellar core regions and their long axis averaged EPAs (as obtained with the Lorentzian fitting procedure) are shown in Figure 1 with black line segments. White lines show intensity contours obtained at levels of 0.5 , $0.6,0.7,0.8,0.9,0.95$, and 0.99 of the peak. Dark lines show identical fractional intensity levels obtained from the Lorentzian fit models. Estimates of the EPA and axis ratio obtained from the fits are displayed in Columns 4 and 5 of Tables 1 and 2, respectively.

In order to quantify the quality of the fits regarding the intensity structures imaged by Herschel, we calculated the linear Pearson correlation parameter (LPCP) of the Lorentzian model and the observed intensity structure for each core. This parameter is given in the last column in Tables 1 and 2 . Figure 2 show scatter plots of the Lorentzian modeled intensity (I(MODEL)) against the $350 \mu \mathrm{m}$ observed emission (I(DATA)). One can see strong correlations between the two intensities (e.g., for cores 1, 3, and 9), lack of correlations (e.g., for cores 4 and 21), and cases in between. The lack of correlation, i.e., a poor fit of the Lorentzian model, is mainly due to complex structure around the central position of the cores or to the presence of a secondary stronger peak nearby. In order to avoid a bias in the forthcoming analysis, we rejected the regions for which the LPCP is lower than $70 \%$. This value provides a good compromise for rejecting ambiguous fits while ensuring that good quality fits are kept. We point out, however, that rejecting the regions for which the LPCP is lower than 55\% would not have affected the general conclusions of this work. We also rejected core 11 , which has an axis ratio of unity from the Lorentzian fit, and so the EPA estimate cannot be trusted for this object. In Figure 1, all rejected cores are marked by a red cross.

The histogram of the distribution of the EPAs for the sample of cores passing the tests discussed above is shown in Figure 3. The standard deviation is minimized for a distribution centered around the median value of $95^{\circ}$, with a standard deviation of $41^{\circ}$. These results are discussed further in Section 5.

\footnotetext{
20 In practice we also assumed and tested Gaussian distributions. The results were similar to those obtained with Lorentzian distributions.

21 http://www.exelisvis.com/docs/mpfit2dpeak.html.
} 

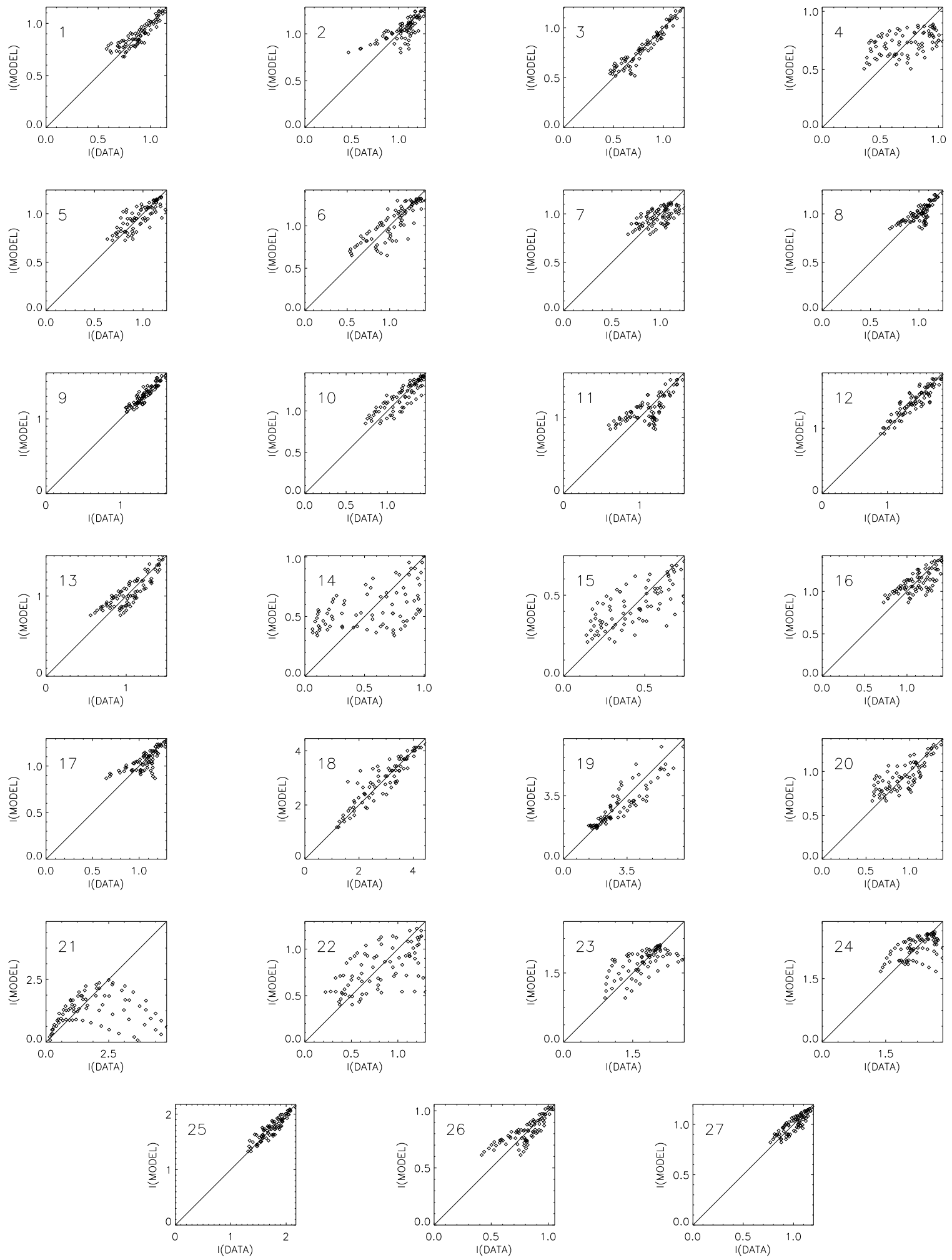

Figure 2. Distribution of the modeled intensity obtained with Lorentzian fits against the Herschel $350 \mu \mathrm{m}$ observed intensity shown for each region displayed in Figure 1. There are 81 pixels shown in these panels. Strong correlations indicate that the Lorentzian model provides a good fit.

\subsection{Histogram of Inferred Magnetic Field Position Angles}

The histogram of inferred magnetic field orientations is obtained by shifting all the measured $350 \mu \mathrm{m}$ polarization angles by an angle of $90^{\circ}$ (Matthews et al. 2014). This histogram is shown in Figure 4. The distribution is strongly peaked, with an average value of $\theta_{\mathrm{B}} \approx 29^{\circ}$ and a standard deviation of $\approx 10^{\circ}$.

\section{STATISTICAL RESULTS}

A visual summary of our results is shown on the Lupus I map displayed in Figure 5. The orientations of the average elongations of the prestellar core structures are shown with black lines. The locations of the cores displayed in Table 2, which are rejected by our analysis, are indicated with white crosses. The 


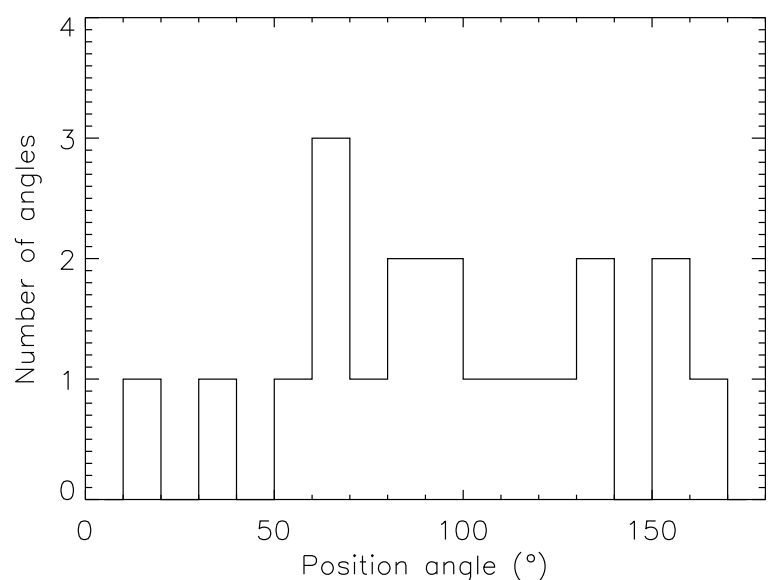

Figure 3. Histogram of the distribution of the EPAs estimated for the list of sources displayed in Table 1 and retained in our analysis, as discussed in Section 4.1 .

inferred projected magnetic field orientations are shown with red lines.

\subsection{Morphology Discussed by Matthews et al.}

The morphology of the main filament running in the Lupus I region from bottom left to top right in Figure 5 has been discussed by Matthews et al. (2014). The relation between the large-scale magnetic field, as probed with starlight polarimetry by Rizzo et al. (1998) in the diffuse ISM surrounding Lupus I, the magnetic field structure probed in denser regions of the filament with the BLASTPol 2010 data, and the Lupus I main filamentary shape observed on large-scale, has also been discussed by these authors. Consistency is found between the mean magnetic field orientation in dense and diffuse regions of the interstellar medium (ISM), and the elongation of the main filament is found on average to be nearly perpendicular to the large-scale magnetic field structure as seen in projection on the POS.

Matthews et al. (2014) investigated the relation between the large-scale magnetic field and an arced filament model that they introduce for more accurately describing the shape of the main filament in Lupus I. This bent filament is parameterized by an arc of a circle centered at R.A. $(\mathrm{J} 2000)=231.77$, decl. $(\mathrm{J} 2000)=-37.67$, with a radius of $=4.92$. With this model, the authors found that the magnetic field orientations probed along high column density regions with submm data, and in the diffuse ISM with optical data, bracket the filament normal, differing from it by 9.8 and 8.6 , respectively.

\subsection{Core Elongation Distribution versus Large-scale Molecular Cloud Structures}

The histogram of the EPAs for the 19 cores passing the analysis test discussed in Section 4.1 is shown in Figure 3. Data for these 19 cores are listed in Table 1.

The standard deviation is minimized for a distribution centered around the median value of $95^{\circ}$, but whether or not we try to minimize this parameter, high standard deviation values of the order of $41^{\circ}$ are obtained, i.e., values quite close to the value of $\approx 52^{\circ}$ expected for a strictly random distribution (Serkowski 1962). Therefore, the EPAs appear to have no special global alignment with respect to the large-scale main filament model discussed by Matthews et al. (2014), nor do they appear to have a special alignment with respect to the pattern of secondary filaments that often run orthogonally to this large-scale main filament.

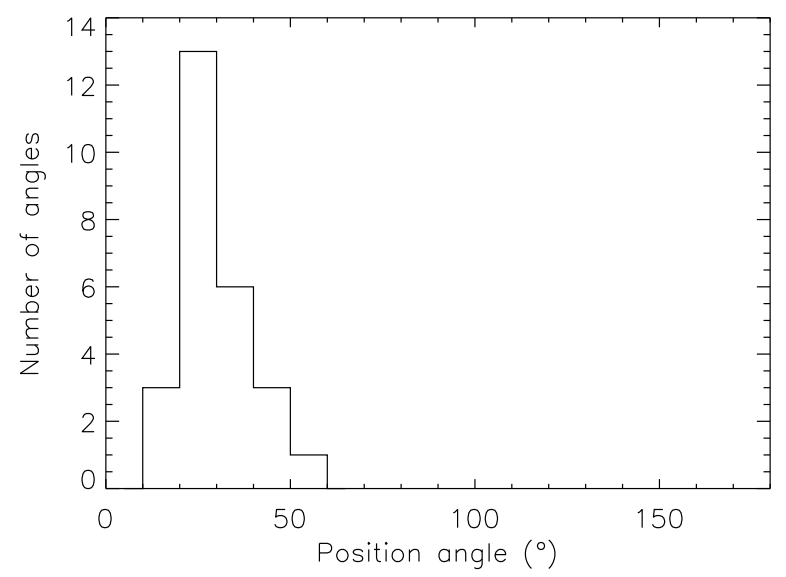

Figure 4. Histogram of the inferred magnetic field orientations, as derived from the BLASTPol $2010350 \mu \mathrm{m}$ polarization data. The inferred field direction is obtained by adding $90^{\circ}$ to the measured polarization angle.

By using the description of the arced filament model proposed by Matthews et al. (2014) we calculated the offset PA between each core's EPA and the local normal to the bent filament. Figure 6 shows the histogram of these PA offsets, obtained for the 19 cores passing the selection of Section 4.1 and listed in Table 1. Here again we find a distribution consistent with random, which means that no specific orientation can be seen between the EPAs and the large-scale bent filament model.

\subsection{Core Elongation Distribution versus Magnetic Field Structures}

Due to problems with a damaged blocking IR filter during flight and resultant systematics, limiting the 2010 data set (as discussed by Matthews et al. 2014), the information provided by the BLASTPol $350 \mu \mathrm{m}$ polarimetry data is pixelized in pixels of size $2.5 \times 2.5$. This corresponds approximately to kernels of $16 \times 16$ pixels in the Herschel $350 \mu \mathrm{m}$ intensity map, i.e., about 3.2 times larger than the regions used for characterizing the core morphology structures. Because of this we decided not to compare the orientation of each individual core to its local magnetic field structure. As a consequence, in the following we only compare the distribution of the elongation of the cores to the mean magnetic field orientation.

The elongation of the main filament is found on average to be nearly perpendicular to the large-scale magnetic field structure as seen in projection on the POS (Matthews et al. 2014). Our result therefore also implies that there is no specific orientation of the average elongation of the cores with respect to the largescale structure of the magnetic field that might shape the main filament.

However, since the structure of the magnetic fields in the high density regions (which have not been probed with submm polarimetry) is not clear, we have searched for possible correlations between the elongations of subsets of cores having POS displacements from submm pseudo-vectors smaller than various threshold values. Given that the mean width of the Lupus I main filament is of the order of 7.5, we first calculated mean and median EPA estimates for cores having displacements of less than 7'.5 from the nearest pseudo-vector. The standard deviation of the distribution of EPAs for this subset is higher than that obtained for the complete set of selected cores, i.e., it too is consistent with a random distribution. When the same calculations are performed for a displacement lower than the size of the BLASTPol 2010 beam $(2.5)$, the size of the sample $(N=6)$ 


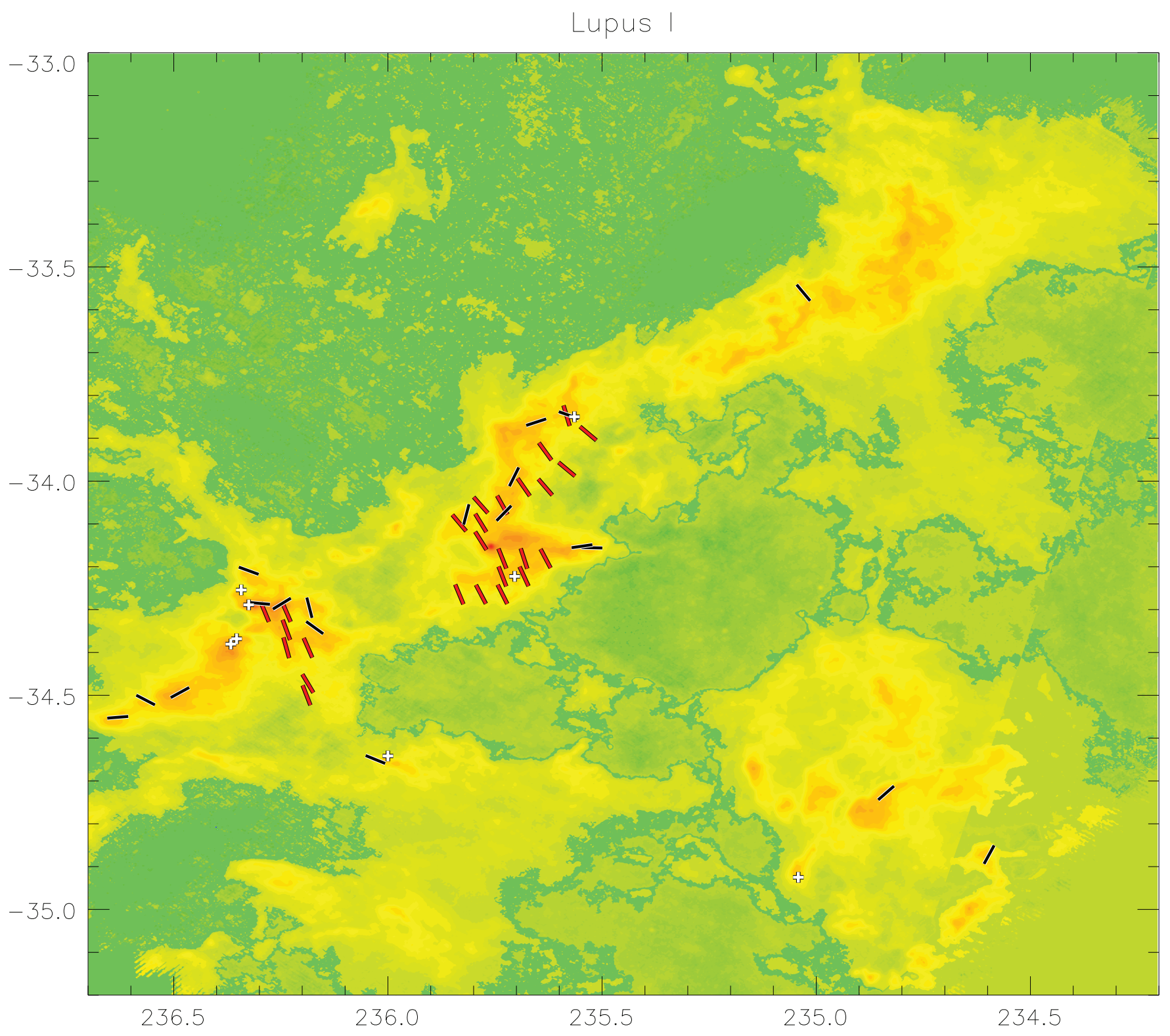

Figure 5. Map showing magnetic field orientations red lines inferred from the BLASTPol $350 \mu \mathrm{m}$ data. The locations of the prestellar cores discussed by Rygl et al. (2013) are shown with black lines (where orientations could be robustly derived) and white crosses (where no clear orientation could be fit). The map in the background shows the Herschel $350 \mu \mathrm{m}$ dust emission intensity map.

(A color version of this figure is available in the online journal.)

starts to be quite small, but the same conclusion can be drawn. As an ultimate test, we calculate a mean EPA of $103^{\circ}$ for the subset of cores matching within a BLASTPol beam. This means there is an average offset angle of about $16^{\circ}$ between the mean short axis direction of the sample of cores and the mean orientation of the magnetic field of $\approx 29^{\circ}$. Although this result is obtained for a sample of two cores only, it is consistent within the uncertainties with the results obtained by Ward-Thompson et al. (2009) and Tassis et al. (2009), but obviously no strong conclusions can be drawn from this. The work by Ward-Thompson et al. (2009) and Tassis et al. (2009) is discussed in the next section.

A summary of all these results is displayed in Table 3.

\section{DISCUSSION}

Our core orientation characterization method is based on 2D Lorentzian fits, which means that no assumptions on whether the 3D structure of the cores is oblate or prolate have been made.
Table 3

Statistics on PA Distributions

\begin{tabular}{|c|c|c|c|c|}
\hline Field & $\begin{array}{c}\text { Sample } \\
\text { Size }\end{array}$ & $\begin{array}{c}\text { Mean } \\
\left({ }^{\circ}\right)\end{array}$ & $\begin{array}{c}\text { Median } \\
\left(^{\circ}\right)\end{array}$ & $\begin{array}{l}\sigma \\
\left(^{\circ}\right)\end{array}$ \\
\hline $\mathrm{EPA}(\text { Cores })^{\mathrm{a}}$ & 19 & 96 & 95 & 41 \\
\hline PA(B-Field) ${ }^{b}$ & 26 & 29 & 28 & 9 \\
\hline $\mathrm{EPA}(\text { Cores })^{\mathrm{c}}$ & 12 & 114 & 108 & 46 \\
\hline $\mathrm{EPA}(\text { Cores })^{\mathrm{d}}$ & 6 & 112 & 108 & 43 \\
\hline $\mathrm{EPA}\left(\right.$ Cores) ${ }^{\mathrm{e}}$ & 2 & 103 & 108 & 7 \\
\hline
\end{tabular}

Notes.

a Average position angle estimate for the sample of cores listed in Table 1.

${ }^{\mathrm{b}}$ Average inferred magnetic field position angle estimate.

c Average EPA for the sample of cores with distance less than 7.5 from a polarization pseudo-vector as seen on the POS.

${ }^{d}$ Same as (c) but for a distance lower than 2.5, similar to the effective beam size of the BLASTPol 2010 data.

${ }^{\mathrm{e}}$ Same as (c) but for a distance less than 1'.25. 


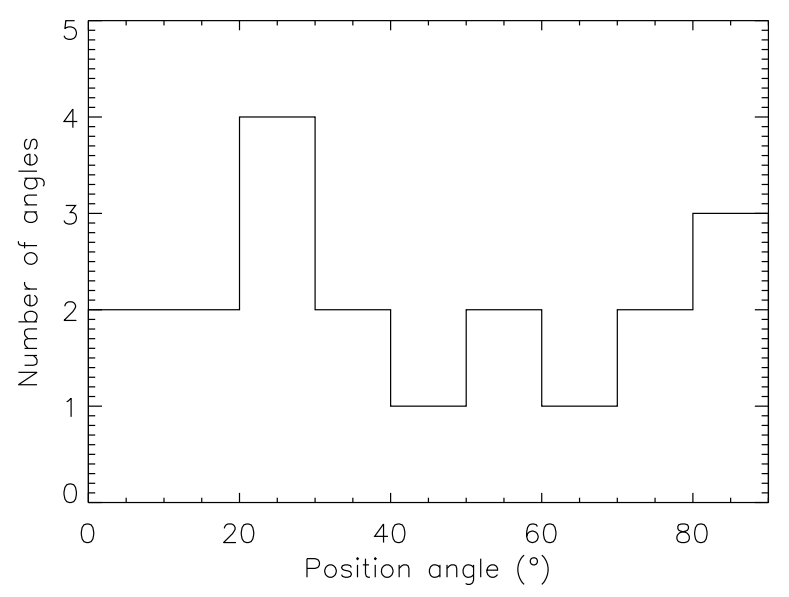

Figure 6. Histogram of the distribution of the offset angles between the core EPAs and the normal to the arced filament model discussed by Matthews et al. (2014).

We chose not to investigate these aspects because, due to the integration of the signal along the LOS, it is not possible to spatially separate the contribution of the dust emission provided by the filament from that originating in the cores. This is particularly the case in crowded regions at different evolutionary stages (see Figure A1 of Rygl et al. 2013) where overlapping cores add confusion. It has been possible, however, to define EPAs for 19 sources from the 27 objects in the sample, as listed in Table 1.

For the remaining targets of the sample listed in Table 2 it was not possible to define EPA values with high confidence. In the case of core 11 , we believe this is a result of projection effects due to the complex three-dimensional (3D) structures of this core. The shape of this object might be that of an oblate disk seen face-on or of a prolate ellipsoid pointing end up, as indicated by the axis ratio of unity. The probability of this is expected to be low (Gammie et al. 2003) but cannot be totally rejected (Tassis et al. 2009), in particular if the amount of dust emission between the observer and a given core is negligible. For prestellar cores $4,7,14,21,22,23$, and 24, the problems with modeling their $2 \mathrm{D}$ projected shapes is more likely coming from the complex dust emission intensity distribution along their LOSs, in particular in regions containing high numbers of cores with embedded sources (Rygl et al. 2013).

With all the limitations mentioned above and the results discussed previously, the general picture emerging from our analysis is that the sample of selected prestellar cores surviving our analysis looks quite randomly oriented on the POS, and therefore randomly oriented with respect to the main filament of the Lupus I molecular cloud, as well as with respect to the mean magnetic field structure probed in various density regimes in this region. This last finding is in agreement with the analysis of simulated cores (or clumps) provided by Gammie et al. (2003). These authors study the formation of 3D analogs of cores using self-consistent, time-dependent numerical models of molecular clouds. Their models include decay of initially supersonic turbulence in an isothermal, self-gravitating, magnetized fluid. All simulated cores are not expected to be self-gravitating and their axes are not strongly aligned with the large-scale magnetic field.

Matthews et al. (2014) suggest a correlation between the main shape of the filament and the mean magnetic field on large scales, but secondary filaments are also observed, which make the picture of Lupus I a complex one once smaller scales

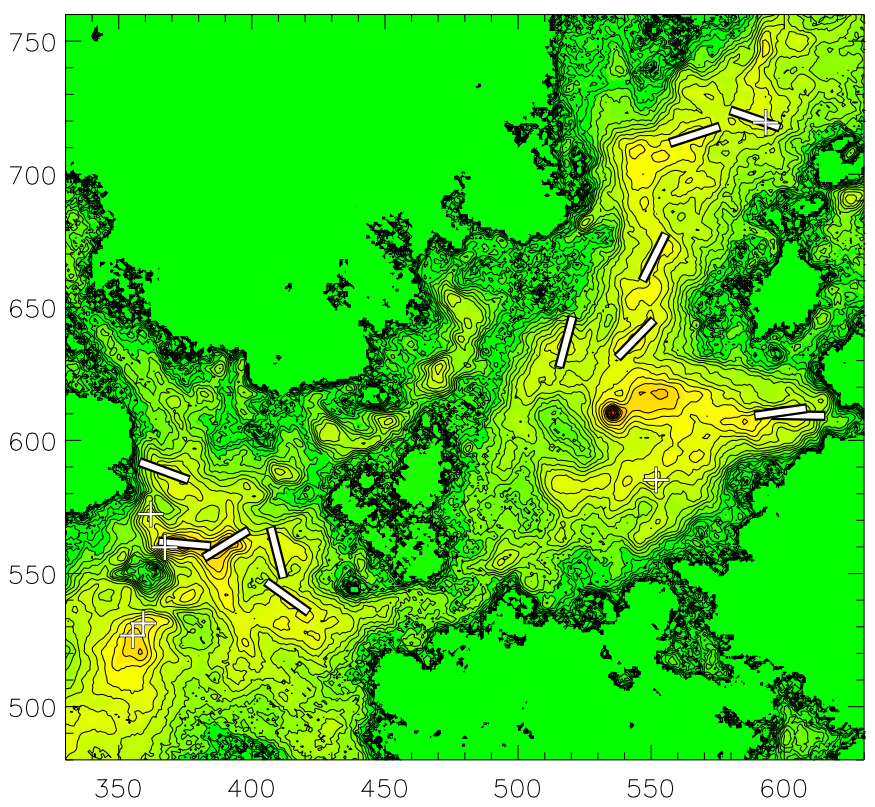

Figure 7. Zoom in on the region centered around R.A. $(J 2000)=236.0$, decl. $(\mathrm{J} 2000)=-34.2$ where the magnetic field has been probed with BLASTPol in Lupus I (see Figure 5). Contour lines showing spatial variations of the column density have been overlaid for comparison with the core elongations (shown with white lines) with respect to the $2 \mathrm{D}$ morphology of the cloud structures in their neighborhood.

(A color version of this figure is available in the online journal.)

are considered. To illustrate this aspect, we plotted in Figure 7 intensity contours showing spatial variations of the column density overlaid on the core elongations (shown with black lines). In this part of the Lupus I cloud, a secondary filament crosses the main cloud filament, as discussed by Matthews et al. (2014), near where the magnetic field has been probed by BLASTPol (see Figure 5). It is clear from this figure that the cloud sub-structure is complex, so that the morphology of the cores may be determined by their local environment, i.e., the local physics of the filament, rather than by the large-scale morphology of the filament.

Regarding the effects of the magnetic fields on sub-parsec scales, various studies (e.g., Hildebrand et al. 1999) have shown that a "polarization hole," or in other words, a decrease of the polarization fraction as a function of the intensity, is measured toward the majority of molecular cloud cores observed with submm polarimetry. Therefore, it is not yet clear whether or not magnetic fields are probing deep into the cores, in particular above visual extinction of about $10 \mathrm{mag}$, where dust grain alignment might be inefficient (Lazarian 2007; Pelkonen et al. 2009). Apparent depolarization may also occur because of a lack of angular resolution, the effect of which is to smooth complex small-scale magnetic fields structures thereby producing a net low degree of polarization. A mean offset of $\approx 30^{\circ}$ between the short axis of cores embedded in six distinct Bok Globules and the magnetic field orientation in their local diffuse environment was discovered by Ward-Thompson et al. (2000, 2009). Similar results have been subsequently obtained by Tassis et al. (2009) for a sample of 24 molecular clouds. The latter study concentrates on high-mass molecular cloud cores that are larger and denser regions than Bok Globules, but these authors also find that the magnetic field orientation is close to the shortest cloud axis by showing on average a deviation of $24^{\circ}$ 
Alignment of density structures with respect to magnetic fields has also been studied on smaller scales, with mixed results. For low-mass cores, Hull et al. (2013) find no alignment of outflows (and thus, presumably, disks) with respect to corescale magnetic fields, while Chapman et al. (2013) do find evidence for such an alignment. For high-mass cores, Poidevin \& Bastien (2006) show that magnetic fields wrapped up by envelope rotation can be expected. Thus, the subject remains open to more investigation.

We expect that the BLASTPol 2012 data collected during 2012 December-2013 January, currently under analysis, may be able to address these issues.

\section{SUMMARY}

In this work we first calculated the average EPAs for a sample of prestellar cores identified by Rygl et al. (2013). We then compared the distribution of the core EPA values to the mean shape of the large-scale filaments in Lupus I.

The average orientation of the cores, as seen on the POS, is obtained by fitting 2D Lorentzian models to the $350 \mu \mathrm{m}$ Herschel dust emission intensity map centered at the position of the prestellar sources.

We find the EPAs to be consistent with a random distribution, which means no specific orientation of the morphology of the cores is observed with respect to a large-scale filament shape model for Lupus I. Similar results are found when the average elongation of each core is compared to the closest normal of a large-scale bent filament model discussed by Matthews et al. (2014).

As a second step we compared this distribution with the mean orientation of the magnetic fields probed with $350 \mu \mathrm{m}$ polarimetry in the high density regions of Lupus I with the BLASTPol experiment. Here again we do not find any correlation with respect to the large-scale magnetic field structure.

Our main conclusion is that the local filament dynamicsincluding secondary filaments that often run orthogonally to the primary filament - and possibly small-scale variations in the local magnetic field direction could be the dominant factors to explain the final orientation of each core.

The BLASTPol collaboration acknowledges support from NASA (through grant Numbers NAG5-12785, NAG5-13301, NNGO-6GI11G, NNX0-9AB98G, and the Illinois Space Grant Consortium), the Canadian Space Agency (CSA), the Leverhulme Trust through the Research Project Grant F/00 407/BN, Canadas Natural Sciences and Engineering Research Council (NSERC), the Canada Foundation for Innovation, the Ontario Innovation Trust, the Puerto Rico Space Grant Consortium, the Fondo Institucional para la Investigación of the University of
Puerto Rico, and the National Science Foundation Office of Polar Programs. C.B.N. also acknowledges support from the Canadian Institute for Advanced Research. Finally, we thank the Columbia Scientific Balloon Facility (CSBF) staff for their outstanding work. F.P. also thanks the Spanish Ministry of Economy and Competitiveness (MINECO) under the Consolider-Ingenio project CSD2010-00064 (EPI: Exploring the Physics of Inflation) for its support.

\section{REFERENCES}

Ballesteros-Paredes, J., Hatmann, L. W., Vásquez-Semadeni, E., Heitsch, F., \& Zamora-Avilés, M. A. 2011, MNRAS, 411, 65

Bonnell, I. A., Dobbs, C. L., \& Smith, R. J. 2013, MNRAS, 430, 1790

Chapman, N. L., Davidson, J. A., Goldsmith, P. F., et al. 2013, ApJ, 770, 151

Falceta-Gonçalves, D., Lazarian, A., \& Kowal, G. 2008, ApJ, 679, 537

Gammie, C. F., Lin, Y.-T., Ostriker, E. C., \& Stone, J. M. 2003, ApJ, 592,203

Girart, J. M., Frau, P., Zhang, Q., et al. 2013, ApJ, 772, 69

Hara, A., Tachihara, K., Mizuno, A., et al. 1999, PASJ, 51, 895

Heitsch, F., Stone, J. M., \& Hartmann, L. W. 2009, ApJ, 695, 248

Hildebrand, R. H., Dotson, J. L., Dowell, C. D., Schleuning, D. A., \& Vaillancourt, J. E. 1999, ApJ, 516, 834

Hull, C. L. H., Plambeck, R. L., Bolatto, A. D., et al. 2013, ApJ, 768, 159

Johnstone, D., Wilson, C. D., Moriarty-Schieven, G., et al. 2000, ApJ, 545,327

Kirk, H., Johnstone, D., \& Di Francesco, J. 2006, ApJ, 646, 1009

Lazarian, A. 2007, JQSRT, 106, 225

Leão, M. R. M., de Gouveia Dal Pino, E. M., Santos-Lima, R., \& Lazarian, A. 2013, ApJ, 777, 46

Lombardi, L., Lada, C. J., \& Alves, J. 2008, A\&A, 480, 785

Matthews, T. G., Ade, P. A. R., Angilè, F. E., et al. 2014, ApJ, 784, 116

McKee, C. F., \& Ostriker, E. C. 2007, ARA\&A, 45, 565

Molinari, S., Bally, J., Glover, S., et al. 2014, arXiv:1402.6196

Moncelsi, L., Ade, P., Angilè, F. E., et al. 2014, MNRAS, 437, 2772

Nakamura, F., \& Li, Z.-Y. 2011, ApJ, 740, 36

Ostriker, E. C., Stone, J. M., \& Gammie, C. F. 2001, ApJ, 546, 980

Pascale, E., Ade, P. A. R., Angilè, F. E., et al. 2012, Proc. SPIE, 8444, 15

Pelkonen, V.-M., Juvela, M., \& Padoan, P. 2009, A\&A, 502, 833

Planck collaboration, Ade, P. A. R., Aghanim, N., et al. 2011, A\&A, 536, A23

Poidevin, F., \& Bastien, P. 2006, ApJ, 650, 945

Poidevin, F., Falceta-Gonçalves, D., Kowal, G., De Gouveia Del Pino, E., \& Magalhães, A. M. 2013, ApJ, 777, 112

Rizzo, J., Morras, R., \& Arnal, E. 1998, MNRAS, 300, 497

Rygl, K. L. J., Benedettini, M., Schisano, E., et al. 2013, A\&A, 549, L1

Schneider, N., André, Ph., Könyves, V., et al. 2013, ApJL, 766, L17

Serkowski, K. 1962, in Advances in Astronomy and Astrophysics, ed. Z. Kopal (New York: Academic), 290

Tassis, K., Dowell, C. D., Hildebrand, R. H., Kirby, L., \& Vaillancourt, J. E. 2009, MNRAS, 399, 1681

Tassis, K., Talayeh, H., \& Willacy, K. 2012a, ApJ, 760, 57

Tassis, K., Willacy, K., Yorke, H. W., \& Turner, N. J. 2012b, ApJ, 754, 6

Tassis, K., Willacy, K., Yorke, H. W., \& Turner, N. J. 2012c, ApJ, 745, 68

Ward, R. L., Wadsley, J., \& Sills, A. 2014, MNRAS, 439, 651

Ward-Thompson, D., Kirk, J. M., Crutcher, R. M., et al. 2000, ApJ, 537,135

Ward-Thomspon, D., Sen, A. K., Kirk, J. M., \& Nutter, D. 2009, MNRAS, 398,394 